UCRL-JC-113401

PREPRINT

\title{
Phonons and Metastability in Compressed Nitrogen
}

\author{
T. W. Barbee III
}

This paper was prepared for submittal to the proceedings for the Joint AIRAPT/APS Conference

June 28 - July 2, 1993

Colorado Springs, $\mathrm{CO}$

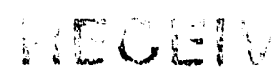

August 1993

\section{SEP 031993}

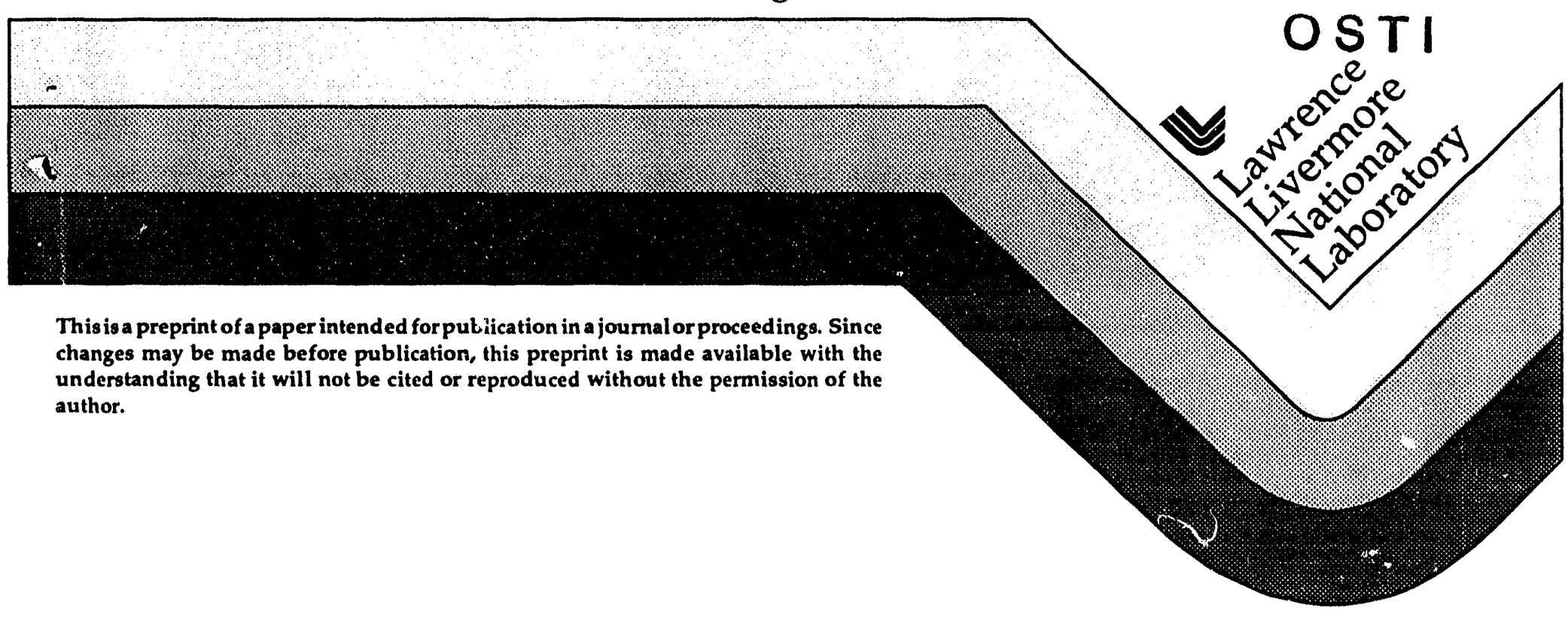




\section{Disclaimer}

Thls document was prepared as an account of work sponsored by an agency of the United States Government. Neither the United States Government nor the University of California nor any of their employees, makes any warranty, express or Implied, or assumes any legal llablitty or responsiblitty for the accuracy, completeness, or usefulness of any Information, apparatus, product, or process disclosed, or represents that its use would not Infringe privately owned rights. Reference herein to any specific commercial products, process, or service by trade name, trademark, manufacturer, or otherwise, does not necessarily constitute or Imply Its endorsement, recommendation, or favoring by the United States Government or the University of Callfornla. The vlews and opinlons of authors expressed hereln do not necessarliy state or refect those of the United States Government or the University of Callfornia, and shall not be used for advertising or product endorsement purposes. 


\title{
PHONONS AND METASTABILITY IN COMPRESSED NITROGEN
}

\author{
T. W. Barbee III \\ Department of Physical Sciences \\ Lawrence Livermore National Laboratory \\ Mail Stop L-299 \\ P.0. Box 808 \\ Livermore, CA 94551
}

The metastability of a recently proposed candidate for a polymeric structure of nitrogen is confirmed by using linear response theory to calculate the phonon dispersion throughout the Brillouin zone. It is found that the structure is mechanically stable for pressures below at least $360 \mathrm{GPa}$. Calculations of Raman and infraredactive phonon frequencies are presented for this metastable phase, and calculations of Raman frequencies for two molecular structures are compared with experimental data.

\section{INTRODUCTION}

At ambient pressure and low temperature, nitrogen freezes into a diatomic solid where strongly covalent ( $N \equiv N$ ) $\mathrm{N}_{2}$ molecules are weakly (van der Waals) bonded to each other. [1] At high pressures, theory predicts dissociation of the molecule and formation of monatomic phases similar to those observed in other group-V elements, i.e., phosphorus and arsenic. At extremely high pressures, nitrogen is predicted to occur in the simple cubic structure. [2] Two calculations $[3,4]$ of the transition pressure for the dissociation of the $\mathrm{N}_{2}$ molecule yielded results below $100 \mathrm{GPa}$ (1 Mbar). However, diamond anvil cell experiments at pressures up to $180 \mathrm{GPa}$ indicate that room-temperature nitrogen remains diatomic to these pressures. $[5,6]$ Conversely, an anomaly observed in a shock compression experiment [7] on fluid nitrogen above $30 \mathrm{GPa}$ has been interpreted [8] as evidence for a transition to a denser atomic phase. These two observations can be reconciled by assuming that a large energy barrier between the molecular and the atomic phases results in high-pressure metastability of the diatomic phase in the room temperature diamond anvil experiments.

Recently, an extensive theoretical study of polymeric phases of nitrogen was performed. [9] A structure with all gauche dihedral angles (the "cubic gauche" (cg) structure) was found to have the lowest total energy of all the polymeric structures considered, but was higher in energy than the molecular structures by approximately $1 \mathrm{eV} /$ atom. The possibility of metastable $c g$ nitrogen was raised by the discovery that a substantial energy barrier existed for the transformation of $c g$ nitrogen into molecular nitrogen at ambient pressure along a path suggested by symmetry considerations.

A stronger requirement for metastability is mechanical stability, i.e., the existence of positive restoring forces for all small motions of the atoms of a crystal about their equilibrium positions. However, mechanical stability provides no information about barrier heights and thus cannot predict the lifetime of the metastable state. An equivalent condition to mechanical stability is the statement that the phonon frequencies $\omega_{Q \lambda}$ for wavevector $q$ and polarization $\lambda$ satisfy $\omega_{Q \lambda}^{2}>0$ throughout the Brillouin zone (BZ), except for the acoustic modes at the zone center.

In this paper, we present ab-initio calculations of phonon spectra $\omega_{q \lambda}$ for nitrogen in two atomic structures at low $(0-12 \mathrm{GPa})$ and high $(240-$ $360 \mathrm{GPa}$ ) pressures using the linear response formal. ism described below and discuss the consequences for metastability. The linear response method is particularly well suited to calculations of complete phonon dispersion relations throughout the Brillouin zone. We find that nitrogen in the $c g$ structure is mechanically stable at both low and high pressures, while nitrogen in the simple cubic structure is mechanically unstable below $240 \mathrm{GPa}$. In addition, we present calculations of the frequencies for zone center modes in $c g$ and molecular nitrogen as a function of pressure to aid in the identification of the these phases.

\section{THEORETICAL METHOD}

The calculations whose results are presented below employ density functional theory within the local 
density approximation ( $\mathrm{Y} \because \ldots)$, with the electron-ion interaction modeled by a pseudopotential. A nonlocal, norm-conserving pseudopotential [10] in a separable form [11] was used in the calculations.

The linear response (LR) nuethod for calculating phonon frequencies has been described in detail elsewhere, [12] so only a brief review will be given here. The starting point of the LR method is a selfconsistent calculation of the electronic wavefunctions in the unperturbed crystal. The crystal is then distorted according to the phonon mode under consideration.

In the distorted crystal, the Hamiltonian, wavefunctions, external (i.e., unscreened electron-ion) potential, and total energy are formally expanded in a perturbation series using the amplitude of the phonon distortion as a small parameter. The first order perturbed wavefunctions can then be obtained from a self-consistent calculation. The primary advantage of the LR method over the frozen phonon [13] method is that the calculations may be performed in the original unit cell rather than in a supercell; hence, the calculations are much faster for long wavelength modes or low symmetry modes.

After self-consistency is reachod, the dynamical matrix $D(q)$ can be found analytically from an expression in which self-consistent quantities only appear in first order. Once the dynamical matrix $D(q)$ is determined, it can be diagonalized to find the harmonic phonon frequencies $\omega_{q \lambda}$ and polarizations $\varepsilon_{q \lambda}$ for all modes $\lambda$ at wavevector $q$.

\section{RESULTS AND DISCUSSION}

The first structure considered was the simple cubic structure for nitrogen, which was recently predicted [2] to be stable for pressures above $1500 \mathrm{GPa}$, but is expected to be unstable at lower pressures. Figure 1 shows the squares of the phonon frequencies $\omega^{2}$ for nitrogen in the simple cubic structure at $240 \mathrm{GPa}$ plotted as a function of wavevector along high-symmetry lines in the BZ. Since the squared phonon frequency $\omega^{2}$ is negative for at least one mode, the structure is mechanically unstable.

The phonon instabilities at certain highsymmetry points in the $\mathrm{BZ}$ indicate possibly more stable structures. The distortion that takes the simple cubic structure into the arsenic (A7) structure is related to the $R$ point phonon, the transformation to the black phosphorus structure is related to the

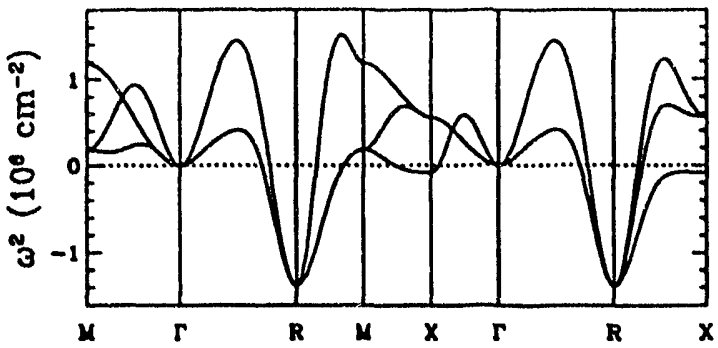

Figure 1. Phonon dispersion for nitrogen in the simple cubic structure at high pressure $(240 \mathrm{GPa})$. The squares of the phonon frequencies $\omega^{2}$ are plotted versus wavevec. tor q. The structure is mechanically unstable, as shown by the existence of phonon modes with $\omega_{a \lambda}^{2}<0$ near the $R$ and $X$ points of the Brillouin zone.

$X$ point phonon, and the distortion that transforms simple cubic to $\mathrm{cg}$ is related to the $\mathrm{M}$ point phonon.

The instabilities near the $R$ and $X$ points sug. gest barrierless paths from simple cubic to the arsenic (A7) and black phosphorus structures, respectively, at $\sim 240 \mathrm{GPa}$. While the M-point frequency is real, a previous study [9] found that the cg structure was more stable (i.e., lower in energy) than A7 at these pressures, which is consistent with an extremely small barrier from simple cubic to $\mathrm{cg}$ which we found in frozen phonon calculations.

The $\mathrm{cg}$ structure consists of a body centered cubic Bravais lattice with a four-atom basis at $(x, x, x)$, $(0.5-x,-x, 0.5+x),(-x, 0.5+x, 0.5-x)$, and $(0.5+x, 0.5-x,-x)$ in Cartesian coordinates. The structure is completely determined by specifying the lattice constant $a$ and the internal parameter $x$. When $x=0$, the cg structure becomes simple cubic with lattice constant $a / 2$.

The phonon dispersion relation for $c g$ nitrogen at $12 \mathrm{GPa}$ is shown in Fig. 2. All phonon modes have positive frequency and no modes appear to be soft, indicating that this structure is mechanically stable.

Figure 3 shows the phonon dispersion relation for cg nitrogen at $360 \mathrm{GPa}$. All phonon modes are still of positive frequency, indicating mechanical stability, although a soft mode is developing at the $N$ and $H$ points, suggesting instability at higher pressures. From Figs. 2 and 3, we conclude that $c g$ nitrogen is metastable at and below $360 \mathrm{CPa}$.

Since the linear response method provides only the curvature of the energy versus displacement curve (i.e., the harmonic phonon frequency), we cannot es. timate the lifetime of the metastable state since no estimate of the barrier height is available from linear 


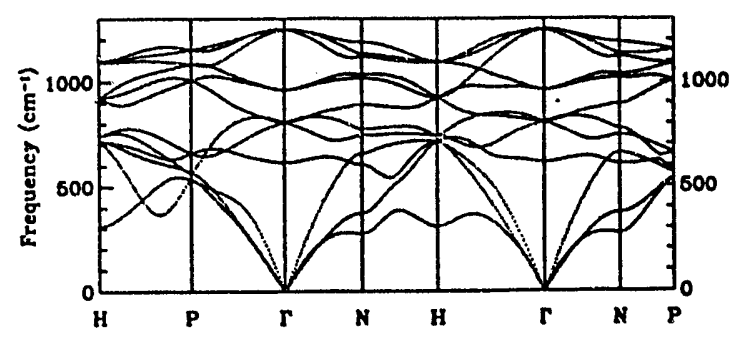

Figure 2. Phonon dispersion $\omega(q \lambda)$ for nitrogen in the cubic gauche structure at low pressure (12 GPa). All phonon modes have real and positive frequency, indicating that the structure is mechanically stable; however, no information about the barrier heights is given (see text).

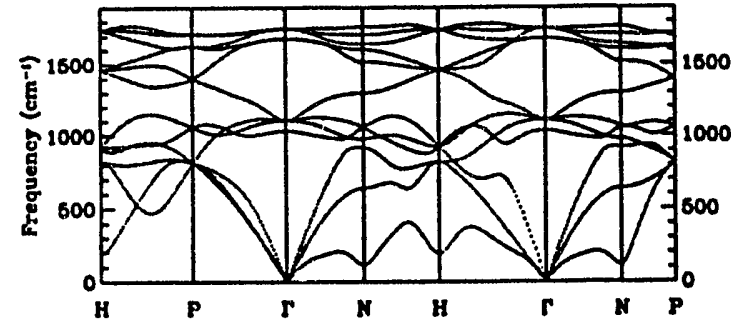

Figure 3. Phonon dispersion $\omega(q \lambda)$ for nitrogen in the cubic gauche structure at high pressure $(360 \mathrm{GPa})$. All phonon modes have real and positive frequency, indicating mechanical stability, although soft modes are developing at the $\mathrm{N}$ and $\mathrm{H}$ points.

response. A previous calculation of barrier heights [9] found a barrier of approximately $0.86 \mathrm{eV} /$ atom for the transformation of $\mathrm{cg}$ nitrogen into the molecular $\beta-\mathrm{O}_{2}$ structure at ambient pressure. For comparison, the barrier to graphitization of diamond is only about $0.3 \mathrm{eV} /$ atom. [14] To determine whether $c g$ nitrogen could be experimentally observed at ambient pressure, the effects of defects and/or surfaces on the metastability should be considered. These could be treated within a supercell, but are probably more amenable to treatment via molecular dynamics using simple interatomic potentials.

Since the most likely route to synthesis of $\mathrm{cg}$ nitrogen will take place in a diamond anvil cell at high pressure and temperature, knowledge of the optical response of $c g$ nitrogen will be necessary to confirm its existence. The $c g$ structure has nine zone-center optical modes. All of these are Raman active, and the two triply degenerate modes are also infrared active. The calculated frequencies of zone-center modes versus pressure are plotted in Fig. 4. The appearance of these modes, together with the disappearance of the

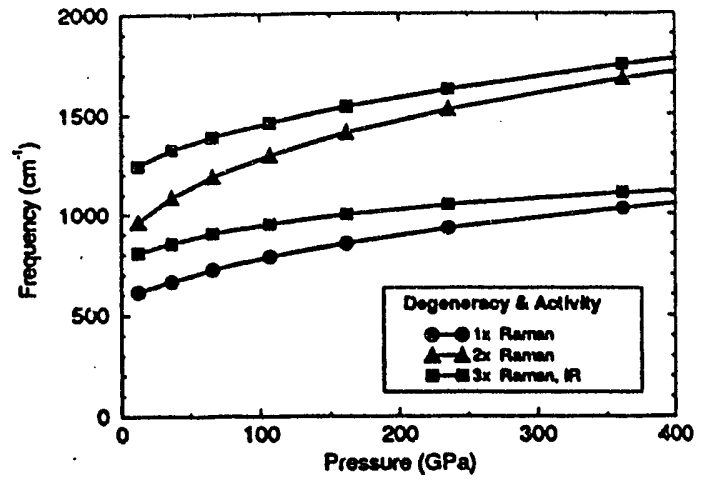

Figure 4. Frequencies of zone center phonon modes in cubic gauche nitrogen as a function of pressure. The lines are guides to the eye. In this structure, all zone center modes are either Raman or infrared active, or both. The degeneracies of the modes are indicated in the legend.

moleculas vibron, would signal the transformation into the $c g$ structure. Since the vibron lies at much higher frequency $\left(\sim 2300-2500 \mathrm{~cm}^{-1}\right.$, depending on pressure), the dissociation of the molecular phase should be clearly observable. The multiply degenerite modes in Fig. 4 may split under non-hydrostatic conditions, so more than four lines may be observed for $c g$ nitrogen.

Calculations of the optically active modes can also help identify the structure of molecular nitrogen at high pressure. We have calculated the zone-center frequencies for two structures of molecular nitrogen: $\alpha-\mathrm{N}_{2}$ (space group $\mathrm{Pa} 3$ ) at low pressure $(<1 \mathrm{GPa}$ ) and $\epsilon-\mathrm{N}_{2}$ (space group $R \overline{3} \mathrm{c}$ ) at $49 \mathrm{GPa}$. In $\alpha-\mathrm{N}_{2}$, the 24 modes at the zone center were calculated using the LR method. The Raman-active frequencies are plotted in Fig. 5 as crosses at a pressure of $1 \mathrm{GPa}$. The experimental low-temperature Raman data [15] for $\alpha$ $\mathrm{N}_{2}$ are shown as small horizontal bars at the left edge of the figure. The calculated vibron frequencies for $\alpha-\mathrm{N}_{2}$ (near $2300 \mathrm{~cm}^{-1}$ ) are about three percent low, while the calculated lattice modes (near $150 \mathrm{~cm}^{-1}$ ) are nearly a factor of two too high in frequency. This could be due to the failure of LDA to describe the molecule-molecule interactions at low density or due to anharmonic effects not included in the LR method.

Our calculation of all 48 zone-center modes for $\epsilon$ $\mathrm{N}_{2}$ at $49 \mathrm{GPa}$ is, to our knowledge, the first such calculation from first principles. The $\mathbf{4 5}$ optical modes all have real and positive frequency, indicating that an energy barrier exists between $R \overline{3} c$ and the closely related $R 3 c$ structure, which has also been proposed 


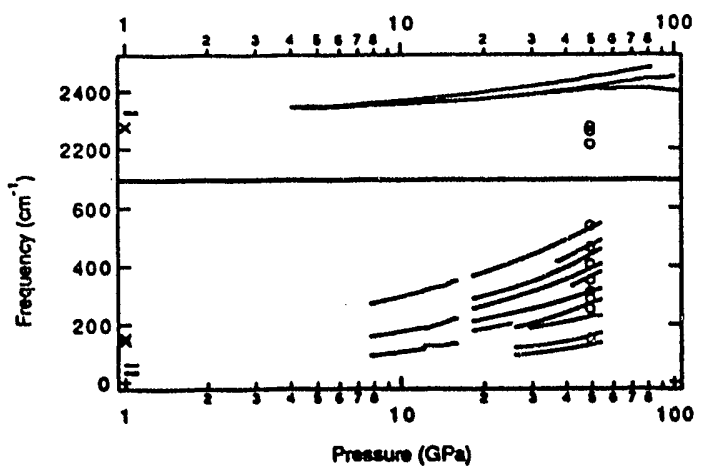

Figure 5. Frequencies of zone center phonon modes in molecular nitrogen as a function of pressure. Lines are experimental data, [15] crosses are calculated frequencies for $\alpha-N_{2}$, and circles are calculated frequencies for $\epsilon-N_{2}$.

for $\mathrm{N}_{2}$ above $24 \mathrm{GPa}$. This is consistent with a previous calculation [16] which found $R \widehat{3} c$ more stable than $R 3 c$ up to $75 \mathrm{GPa}$.

The calculated Raman frequencies are plotted as circles in Fig. 5. Here the vibron frequencies are about seven percent too low, while the lattice modes agree extremely well with experiment, especially if one assumes that some of the calculated modes may be split due to non-hydrostatic conditions in the experiment. We note that the experimental data for $P>4 \mathrm{GPa}$ was taken at room temperature and our calculations assume zero temperature, but the differences between experiment at $15 \mathrm{~K}$ and room temperature are small. [15] Except for the disagreement in the vibron frequencies, the calculated Raman frequencies for $\epsilon-\mathrm{N}_{2}$ agree very well with the experimental data, raising the possibility that $\epsilon-\mathrm{N}_{2}$ persists above $24 \mathrm{GPa}$. We are currently calculating the Raman active modes for several other candidate phases over a range of pressures in order to constrain the possible structures in the 20-100 GPa range.

\section{SUMMARY}

We have studied the possibility of metastable nitrogen in the simple cubic and $c g$ structures via $a b$ initio full-zone phonon calculations using the linear response formalism. The simple cubic structure was found to be mechanically unstable below $240 \mathrm{GPa}$, while the $c g$ structure was found to be metastable up to $360 \mathrm{GPa}$. Calculations of Raman modes for $\epsilon-\mathrm{N}_{2}$ indicate that this structure may persist up to pressures of $50 \mathrm{GPa}$. Further calculations of Raman frequencies for different molecular structures will aid in the determination of the high-pressure phase diagram for nitrogen.

\section{ACKNOWLEDGMENTS}

I gratefully acknowledge conversations with A. McMahan, C. Mailhiot, and H. Lorenzana. This work was performed under the auspices of the U.S. Department of Energy by Lawrence Livermore National Laboratory under Contract No. W-7405-Eng-48, and has been supported in part by the Joint DoD/DOE Munitions Technology Development Program.

\section{REFERENCES}

[1] D. A. Young, Phase Diagrams of the Elements (University of California Press, Berkeley, 1991).

[2] S. P. Lewis and M. L. Cohen, Phys. Rev. B 46, 11117 (1992).

[3] A. K. McMahan and R. LeSar, Phys. Rev. Lett. 54, 1929 (1985).

[4] R. M. Martin and R. J. Needs, Phys. Rev. B 34, 5082 (1986).

[5] R. Reichlin et al., Phys. Rev. Lett. 55, 1464 (1985).

[6] P. M. Bell and H. K. Mao, Physica B+C 1398140B, 16 (1986).

[7] H. B. Radousky et al., Phys. Rev. Lett. 57, 2419 (1986).

[8] M. Ross, J. Chem. Phys. 86, 7110 (1987).

[9] C. Mailhiot, L. H. Yang, and A. K. McMahan, Phys. Rev. B 46, 14419 (1992).

[10] N. Troullier and J. L. Martins, Phys. Rev. B 43, 1993 (1991).

[11] L. Kleinman and D. M. Bylander, Phys. Rev. Lett. 48, 1425 (1982).

[12] S. Baroni, P. Giannozzi, and A. Testa, Phys. Rev, Lett. 58, 1851 (1987); 59, 2662 (1987); X. Gonze and J. P. Vigneron, Phys. Rev. B 38, 13120 (1989); 44, 3494(E) (1991); P. Giannozzi, S. Degironcoli, P. Pavone, and S. Baroni, Phys. Rev. B 43, 7231 (1991).

[13] P. K. Lam, M. M. Dacorogna and M. L. Cohen, Phys. Rev. B 34, 5065 (1986).

[14] S. Fahy, S. G. Louie, and M. L. Cohen, Phys. Rev. B 34, 1191 (1986); 35, 7623 (1987).

[15] J. K. Kjems and G. Dolling, Phys. Rev. B 11, 1639 (1975); H. Schneider et al., J. Chem. Phys 96, 8040 (1992); R. Reichlin et al., Phys. Rev. Lett. 55, 1464 (1985).

[16] R. LeSar, J. Chem. Phys. 81, 5104 (1984). 

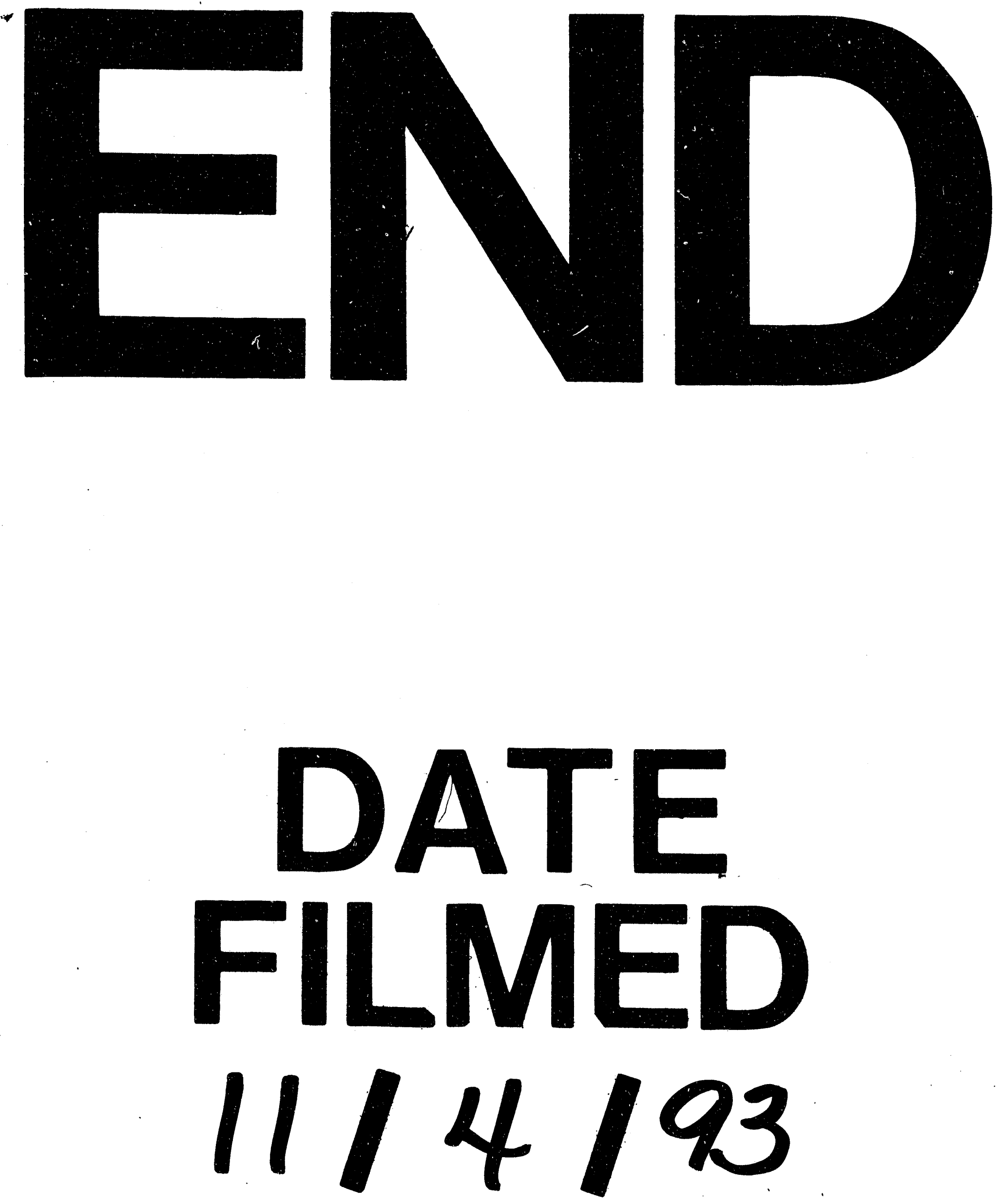
